\title{
1 Children are sensitive to norms of giving
}

2

3 Katherine McAuliffe ${ }^{1,2^{*}}$, Nichola J Raihani ${ }^{3}$ and Yarrow Dunham ${ }^{2}$

4

$5 \quad{ }^{1}$ Department of Psychology, Boston College, Chestnut Hill, MA

$6 \quad{ }^{2}$ Department of Psychology, Yale University, New Haven, CT

$7 \quad{ }^{3}$ Department of Experimental Psychology, University College London, London, United Kingdom

8

$9{ }^{*}$ Corresponding author: katherine.mcauliffe.2@bc.edu 


\section{Abstract}

11 People across societies engage in costly sharing, but the extent of such sharing shows striking cultural 12 variation, highlighting the importance of local norms in shaping generosity. Despite this acknowledged role 13 for norms, it is unclear when they begin to exert their influence in development. Here we use a Dictator 14 Game to investigate the extent to which 4- to 9-year-old children are sensitive to selfish (give $20 \%$ ) and 15 generous (give 80\%) norms. Additionally, we varied whether children were told how much other children 16 give (descriptive norm) or what they should give according to an adult (injunctive norm). Results showed that children generally gave more when they were exposed to a generous norm. However, patterns of compliance varied with age. Younger children were more likely to comply with the selfish norm, suggesting a licensing effect. By contrast, older children were more influenced by the generous norm, yet capped their donations at $50 \%$, perhaps adhering to a pre-existing norm of equality. Children were not differentially influenced by descriptive or injunctive norms, suggesting a primacy of norm content over norm format. Together, our findings indicate that while generosity is malleable in children, normative information does not completely override pre-existing biases.

\section{Keywords}

Generosity, cooperation, dictator game, norms, social cognitive development 
People everywhere engage in costly prosocial behavior, ranging from every-day acts like volunteering time at community events to more tangible gestures like giving away a proportion of one's earnings to charity. Indeed, according to Giving USA, in the last year people in the USA alone gave an estimated $\$ 358.38$ billion dollars to charity (more than $\$ 1,000$ per adult) highlighting the economic importance of understanding the mechanisms supporting generosity in humans.

A great deal of work by economists and psychologists has shown that generous behavior can be readily elicited under laboratory conditions. The most widely used task for capturing generosity in the lab is the Dictator Game (Kahneman, Knetsch, \& Thaler, 1986). In this game, one person - the dictator - is given a sum of money and is asked to allocate the endowment between themselves and a passive recipient. While traditional economic models predict that dictators will keep the entire endowment for themselves because any donation necessarily reduces the dictator's payoff, dictators typically share a portion of their endowment with partners (Engel, 2011). A further striking finding from work on the Dictator Game is that the amount shared with partners shows dramatic variation across different societies (Henrich, Boyd, Bowles, Camerer, Fehr, Gintis et al., 2005). For instance, in one cross-cultural investigation of Dictator Game giving, Hadza participants offered a $20 \%$ share on average, while Tsimane participants typically offered ten percent more (Henrich et al., 2005). In these two societies, few people kept everything for themselves, in contrast to American participants, many of whom refused to share at all (Camerer, 2003). This cultural variation demonstrates that while generosity may be a common human behavior, what constitutes generosity is profoundly shaped by local norms.

An influential approach to addressing how norms affect behavior begins by distinguishing between two different types of normative information, what we refer to here as norm format. Descriptive norms describe what others are doing, while injunctive norms describe what ought to be done to earn social approval (Cialdini, Reno, \& Kallgren, 1990). Descriptive and injunctive norms have garnered a great deal of attention in social psychology and each appears to influence people's behavior in a range of social situations including littering (Cialdini et al., 1990), taking resources from a national park (Cialdini, Demaine, Sagarin, \& Barrett, 2006) and household energy consumption (Schultz, Nolan, Cialdini, Goldstein, \& Griskevicius, 2007). Theoretically, the distinction between descriptive and injunctive norms may be further explored by placing it in a more evolutionarily informed framework, namely by thinking of it as analogous to the distinction between horizontal and oblique transmission (Richerson \& Boyd, 2005; Cavalli-Sforza \& Feldman, 1981). While this framework must be qualified by acknowledging that both descriptive and injunctive norms can, in principle, be transmitted horizontally or obliquely, this can serve as a useful foundation for thinking about which models are most relevant for children at different stages in development. Horizontal transmission, as the name implies, is transmission of information between peers. One central route for horizontal transmission is direct observation of statistical tendencies, i.e., the learning of descriptive norms of who generally does what. Oblique transmission involves acquiring skills and 
knowledge, including explicit rules of conduct, i.e. injunctive norms. Oblique transmission is importantly distinct from vertical transmission, which involves learning specifically from parents. While much early learning happens from parents, children begin to increasingly rely on horizontal and oblique transmission as they age (Hewlett et al., 2011). However, the extent to which children differentially weigh normative information from peers versus adults remains unclear. An emerging picture from developmental work suggests that young children have an initial tendency to rely on adults for social information (Jaswal \& Neely, 2006; Rakoczy, Warneken, \& Tomasello, 2010), which hints at a particular sensitivity to injunctive norms. By contrast, older children are thought to be particularly sensitive to peer influence (Brown, 1990), pointing to the importance of descriptive norms later in development. However, it is not yet know if these same patterns hold when examining children's adherence to norms of generosity.

Some recent work has, however, investigated norm format in the context of adults' giving in the Dictator Game (Bicchieri \& Xiao, 2007; Raihani \& McAuliffe, 2014). Bicchieri and Xiao (2007) found that fair behavior was more affected by participants' expectations of what others give in the dictator game (i.e., a descriptive norm) as opposed to expectations of what ought to be given (an injunctive norm). However, Raihani and McAuliffe's (2014) results did not accord with these findings. They presented dictators with a descriptive norm, an injunctive norm or no normative information. Within norm format, participants saw either a stingy norm (give at least $20 \%$ ) or a generous norm (give at least $50 \%$ ). They found that people were sensitive to both norm content (stingy vs. generous) as well as norm format (injunctive vs. descriptive). Specifically, people gave more when presented with the generous norm than the stingy norm, and injunctive norms increased the propensity to give at least the target amount, whereas descriptive norms did not. Minimally, results from these two studies show that giving behavior is flexible: people's generosity is susceptible to the power of suggestion. However, the extent to which giving behavior is differentially influenced by descriptive versus injunctive norms remains unclear, and in particular, little is known about how or even whether children are sensitive to different norm formats.

Taken together, previous studies of Dictator Game giving in adults have clearly demonstrated that generosity shows natural variation across cultures, and suggest that the transmission of normative information is one potential mechanism by which that variation can be maintained. However, the developmental roots of normative influence are as yet unclear. In particular, it remains possible that children hold strong pre-existing biases, for example towards selfishness (e.g., Benenson, Pascoe, \& Radmore, 2007) that limit the influence of normative information earlier in development. Alternatively, the nature of selfishness versus generosity might itself be open to revision via normative information from early childhood, in which case, children, like adults would be sensitive to locally presented norms. Addressing these issues is critical to understanding how and when cultural variation emerges, as well as how and when we might intervene on developing notions of fairness and generosity.

Broadly past work on children's donations in the Dictator Game demonstrates that, from relatively early in development, children are motivated to share with others, even when doing so comes at a personal 
cost. Children as young as four willingly offer resources to peers across a variety of experimental games (Fehr, Bernhard, \& Rockenbach, 2008; House, Henrich, Brosnan, \& Silk, 2012; Moore, 2009). Of particular relevance here are a number of studies that have examined children's sharing behavior using different versions of the Dictator Game (Benenson et al., 2007; Blake, Corbit, Callaghan, \& Warneken, 2016; Blake \& Rand, 2010; Cowell et al., 2016; Gummerum, Hanoch, Keller, Parsons, \& Hummel, 2010; Rochat et al., 2009; Smith, Blake, \& Harris, 2013). While results from these studies are not entirely consistent, several trends have emerged: First, young children tend to show a selfish bias that is attenuated with age (Beneson et al., 2007; Cowell et al., 2016; Rochat et al., 2009). Second, older children tend to be more equitable than younger children (Fehr et al., 2008; Smith et al., 2013). Third, children rarely give more than an equal split (Benenson et al., 2007; Blake \& Rand, 2010).

In parallel to work on children's emerging prosociality, other work has shown that an understanding of norms is present from early in ontogeny. For instance, Powell and colleagues (2013) showed that even infants expect similar individuals to behave in the same way, suggesting that humans are predisposed to attend to group norms and that these norms guide expectations of how individuals should act. These expectations appear to persist over the course of ontogeny and drive children to intervene to prevent norm violations in others (Rakoczy \& Schmidt, 2012; Rakoczy, Warneken, \& Tomasello, 2008). More specifically, children as young as two years of age respond to norm violations (Rakoczy et al., 2008), and by three children explicitly correct others' behavior (Rakoczy et al., 2008) and are particularly likely to enforce norm violations committed by an in-group member (Schmidt, Rakoczy, \& Tomasello, 2012).

Another recent line of work has begun to investigate how children's understanding of norms influences their prosocial behavior, and begins to explore potential divergences between understanding of what one ought to do (injunctive information) and expectations about what one will do (descriptive information). For instance, Smith and colleagues (2013) showed that American children between the ages of three and eight understand that they ought to share equally and even expect others to share equally with them. Despite this understanding of what they should do, however, it is not until around the age of seven that children actually begin to adhere to this norm of equality. Blake and colleagues (2014) found a similar gap between what children think they should do and what they actually do and showed that children with better self-regulation close this gap faster than those with poor self-regulation. Finally, in a cross-society study of prosocial behavior, House and colleagues (2013) found that children begin to adopt adult-typical patterns of prosocial behavior around middle childhood, suggesting it is not until this period in ontogeny that children begin to internalize their society's norms of generosity. Together, these studies suggest that from early in development children become aware of norms of giving in their respective societies and that they ultimately follow those norms. However, two key questions remain unanswered, and are the focus of the present study. First, does norm format affect children's generosity? That is, will children be differentially influenced by norms about what their peers are doing (descriptive) versus norms about what they ought to do (injunctive)? Second, are children differentially influenced by norms with different 
content, such as those that prescribe selfishness versus generosity? We address these questions by investigating children's costly giving in the Dictator Game. Children are presented with a selfish (give $20 \%$ ) or generous (give $80 \%$ ) norm and we test whether their donations vary in response to these norms relative to their donations in a condition in which they are not given normative information. In addition to varying norm content, we also vary the format of norm that is presented: they either learn what other children do (descriptive norm) or they learn what should be done from the perspective of an authority figure (injunctive norm). Based on past work, we predict that younger children will be less inclined to share than older children. We expect to see this age effect regardless of norm condition, but are particularly interested in whether the content (selfish or generous) or format (descriptive or injunctive) of norms can move children away from their age-typical giving behavior. More specifically, the proposed shift from oblique to horizontal transmission (and so from a reliance on injunctive to descriptive norms) raises the possibility that younger children will be relatively more sensitive to injunctive information than older children, and vice versa for descriptive information. We thus set out to examine children's susceptibility to influence from normative information across early and middle childhood with the aim of clarifying how they are affected by norms that differ in format (descriptive versus injunctive) and content (selfish versus generous)

\section{Methods}

\section{Participants}

We included 268 children. We tested children between the ages of four and nine, as this age group has been successfully tested in the Dictator Game in previous studies and thus allows us to compare our results to past work (Benenson et al., 2007). Children were divided into three age groups: $4 \& 5(\mathrm{~N}=90$, Mean (months) \pm Standard deviation $=60.4 \pm 6.8,47$ Females $(\mathrm{F})), 6 \& 7(\mathrm{~N}=94,82.6 \pm 7.3,48 \mathrm{~F})$ and $8 \& 9(\mathrm{~N}=$ $84,107.4 \pm 7.2,41 \mathrm{~F})$. For ten of our participants we were missing a precise age due to incomplete birthdate information. For those children, ages were estimated from other information on the consent form or from conversations with the child $(\mathrm{N}=3)$, or children were estimated to be their age in years plus six months ( $\mathrm{N}$ =7). We recruited participants in parks, museums, festivals and a lab in New Haven, CT. We received informed consent from parents and assent from children prior to participation. Fifty-five additional children were tested but excluded from the final sample because they failed comprehension (9) or manipulation (33) checks (see Procedure for details), due to experimenter error (9) or due to parental interference (4). Twenty-four additional children participated but were excluded prior to testing based on pre-specified criteria (e.g. non-English speakers, outside of age range, did not like food resource used). They participated because we wanted to give any interested child the chance to play the game. Our sample size determination was based on a pre-specified target of 20 children per each of 15 design cells. We continued to add children to cells when other children were excluded for the pre-specified criteria described above and exceeded our exact target because we continued to test when we had additional time on-site at a testing session. 
171 Children played a one-shot anonymous Dictator Game with an imaginary peer. We used a between-subject design in which children were assigned to one of three norm conditions: injunctive, descriptive or a control in which no norm information was presented (see Table S1 for sample breakdown). Within the injunctive and descriptive conditions, children were assigned to one of two treatments: a selfish norm treatment (give 2 out of 10) or a generous norm treatment (give 8 out of 10 ).

\section{Procedure}

We based our procedure on previous Dictator Game methods that have been successfully employed with children in this age range (Blake et al., 2014; Blake \& Rand, 2009). Participants were introduced to the rewards being used (Starburst candies), asked if they liked them, and given a choice between playing with two different colors. If a child had never tried a Starburst, they were given the opportunity to do so in order to ensure that they were motivated by the resource. Children were then given ten candies and told that they were theirs to keep and that they could do whatever they wanted with them. By assigning ownership in this way we were able to ask about children's generosity. That is, their willingness to share their own resources. The candies were counted out in front of the child in two rows of five. They were then introduced to two paper bags: one bag was for them and one bag was for another child who would be coming to play later. It was explained that they could keep as many candies as they wanted and they could give as many as they wanted to the other child. They were then introduced to one of the condition/treatment combinations or were tested in the no-norm control condition. In the injunctive condition the experimenter said: "I think you should give $2 / 8$ to the other child". In the descriptive condition, the experimenter said: "most kids that play this game give $2 / 8$ to the other child". To make the divisions more salient to the child, the experimenter physically moved the candies on top of the child's bag and the recipient's bag to demonstrate the giving norms. The candies were then placed back in front of the child in two rows of five. In the control, no information about donation behavior was described. Children were then introduced to a "privacy box", a large cardboard box that sat over the study area such that the child could make their decision out of view. Children were also explicitly told that no one would see them make their decision.

To ensure that children understood all aspects of the game, they were asked four comprehension questions before making their decision about how to divide the candy. They were asked (1) to whom the candies belonged; (2) to identify their paper bag; (3) to identify the paper bag of their partner; and (4) whether anyone could see them in the privacy box. The majority of children spontaneously passed these checks $(89 \%, 91 \%, 98 \%, 95 \%)$. If a child did not answer correctly, they were given another explanation and the comprehension question was re-asked. If children continued to fail these pre-decision comprehension checks, they were excluded (see above for number of excluded children). In three cases, the experimenter re-explained but forgot to re-ask a comprehension question. Given that children overwhelmingly passed comprehension checks after the second explanation, these children were included 
in the final sample. After making their decisions, the children were again asked to identify their bag and asked what would happen to the candies in the partner's bag. Because these questions were asked after children had made their decisions, they were not used as exclusion criteria.

To ensure that children had attended to the norm information that we provided, children in injunctive and descriptive conditions were asked to remember the norm content (selfish: give 2 or generous: give 8) before taking their candies home. Children who did not pass this manipulation check were excluded from the sample (see above). Because this entailed rejecting children from these conditions at a higher rate than the control (which did not have a manipulation check), we ran additional analyses that included the children who failed the manipulation check. Including these children did not change the overall pattern of results (see Table S2). Following these checks, children were debriefed: it was explained that another child would be coming but might not get the specific candies that had been donated and that the norm information was not necessarily true.

Early in the study we made a minor change to the script. Notably, experimenters had initially followed the norm manipulation described above with the phrase "that's just what I think you should do (injunctive) / others are doing (descriptive)." This was done to ensure that children understood that they were free to donate however many they wanted (i.e., so that they did not view the norm as a command). However, we ultimately determined that this could weaken the normative content and so removed this phrase, instead, emphasizing that they were free to make whatever decision they wanted. To ensure that this change did not affect our results, we confirmed that our pattern of our results holds regardless of whether these initial sessions are included or excluded (Table S6).

\section{Coding and analysis}

Data were collected live. If parents provided video consent and the child provided assent ( $90 \%$ of sessions), a recording was also taken of the task up until the child made a decision. When the child was about to make a decision in the privacy box, the camera was turned off. After the child made a decision, their bag was saved and the number of candies inside was counted after they left. The experimenter entered the live data and, where possible, the experimental videos were later checked to ensure that no experimenter errors had been made and to double-check comprehension checks. Because children made their decisions in secret, we do not have video confirmation of their allocation decisions. However, because recording their decisions required simply counting between zero and ten candies, errors were unlikely to be introduced at this stage and any errors that did occur should have been equally distributed across conditions.

We performed analyses using three main approaches. We first asked whether average donations varied across experimental conditions and treatments. Specifically, we asked (1) whether children were differentially responsive to injunctive or descriptive norms and (2) whether their responsiveness differed based on receiving generous versus selfish norm content. Because children in the control condition did not receive norm information and so could not contribute to a treatment by condition interaction (the main 
predictor of interest for these analyses), this analysis focused only on children in the two test conditions. To examine whether donations were predicted by condition and treatment, we ran linear models with subject donation ( 0 to 10 candies) as the response term and condition (norm format: descriptive, injunctive, control), treatment (norm content: selfish, generous), age group (4\&5, 6\&7, 8\&9) and gender fit as fixed effects (see Table 1 for information about base levels). Gender was not found to be a significant predictor in any of our models. Analyses were also run with age fit as a continuous effect and this did not change the overall pattern (see SOM for model output from these analyses). Where appropriate based on the analysis at hand, we included interactions between condition, treatment and age group. To determine which factors were important predictors of donations we eliminated single terms from a full model using the 'drop1' command. In this way, we assessed whether their inclusion improved model fit using likelihood ratio tests (LRTs). Based on these tests, we created a minimal model, which included the combination of factors that provided the best fit to our data.

We next collapsed data across injunctive and descriptive conditions and tested whether children were differentially influenced by information about selfish and generous target donations. Here we go beyond an analysis of mean donations to instead examine the distributions of donations in each condition. The distributional analysis has the advantage of allowing us to distinguish between different possible patterns of responding with similar means. For example, an analysis of means might not allow us to distinguish between some children showing large shifts and others not shifting at all, on the one hand, and many children shifting slightly on the other hand (because these two patterns could involve identical mean donations). That is, this analysis supplements the analysis of means by providing insight into the distribution of responses in each condition. We examined distributions within each age group using Kolmogorov-Smirnov tests. We used bootstrapped Kolmogorov-Smirnov tests (using the ks.boot command in the "Matching" package; Sekhon, 2011) because donation data were not perfectly continuous and contained ties.

Finally, we investigated exact compliance to specific norms (i.e., the possibility that a given norm would lead children to give exactly 2 of 10 or 8 of 10, depending on condition, or that children would give exactly 5, conforming to a pre-existing norm of equality) using generalized linear models (GLMs) with norm compliance fit as a binary outcome (complied $=1$, did not comply $=0$ ). To assess the importance of different predictors, we again used LRTs as described above. Again, additional models were run with age fit as a continuous predictor with qualitatively similar results (see SOM). To assess whether compliance varied within particular age groups and treatments, we compared the frequency of children who complied with a given norm (Give 2, Give 8, Give half) in the treatments versus the frequency of children who made those same donations in the control condition. To do this we conducted Pearson Chi Square tests with Yates correction. These were used as an alternative to GLMs because output from our initial GLMs on the subsetted data indicated a convergence issue, most likely due to too few observations for certain factor combinations. All analyses were performed using R software (version 3.1, R Core Team, 2014). 


\section{Results}

Did norms affect children's donations?

The norm format (condition: injunctive vs. descriptive) had no effect on children's donations (Fig. 1). We found no effect of condition on donations (LRT, $p=0.8$ ), no interaction between condition and treatment ( $p$ $=0.8)$ and no three-way interaction between condition $\mathrm{x}$ age $\mathrm{x}$ treatment $(p=0.9)$ when we examined children who had been tested in the two test conditions. Consequently, we collapsed our data across injunctive and descriptive conditions in order to test whether children's donations were differentially affected by the generous and selfish target amounts when compared to the control. Our linear model showed that both age group (LRT, $\chi_{2}^{2}=80.1, p<0.001$ ) and treatment (LRT, $\chi_{2}^{2}=89.6, p<0.001$ ) were significant predictors of donations: older children tended to be more generous than younger children and children in the generous treatment gave more than children in the selfish treatment or control. Children in the selfish condition tended to give less than those in the control $(\beta=-0.6)$, but this was not a significant effect in our minimal model ( $p=0.07$; Table 1). There was no interaction between age group and treatment $(p=0.2)$.

To more directly compare patterns of children's donations in each treatment relative to the control, we examined the relationship between age and donations specifically within the selfish and generous treatments (Fig. 1) by subsetting our data into two sets: (1) children in the selfish and control conditions and (2) children in the generous and control conditions. We then modeled donations as a function of age, treatment and the interaction between the two. Our first model examined children's donations in the selfish vs. control condition and showed that children who received the selfish norm tended to give less than those in the control (Table S4). We also found that younger children were especially likely to show this distinction (LRT, age $\mathrm{x}$ treatment interaction, $\chi_{2}^{2}=19.47, p=0.044$ ). Our second model examined children's donations in the generous vs. control treatments. We found no interaction between age and treatment $(p=0.2)$. However, our reduced model showed that the oldest children gave more than the youngest children (LRT, age, $\chi_{2}^{2}=33.91, p=0.019$; Table S4) and that children donated more in the generous condition compared to the selfish condition (LRT, treatment, $\chi_{1}^{2}=18.20, p=0.038$; Table S4). 


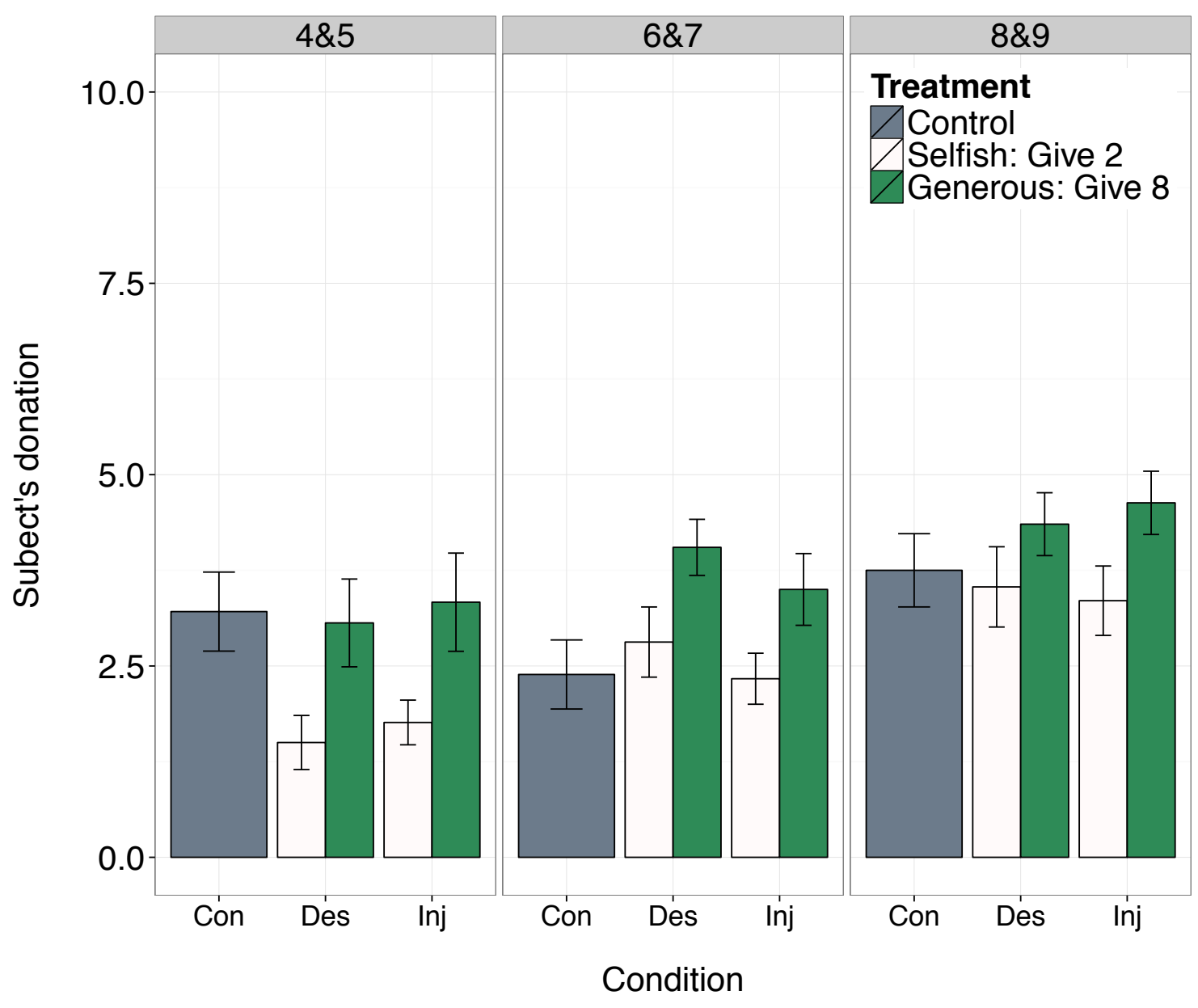

Figure 1. Mean donations of candies (out of ten) in an anonymous Dictator Game. Donations are shown by control (Con), descriptive (Des) and injunctive (Inj) conditions treatment, faceted by participant age group. Within the two norm conditions, children were either exposed to a selfish or generous norm. We used a between-subject design in which children were tested in a single condition/treatment combination. Children in the control condition received no treatment, as the control was run to measure baseline donations by age. Error bars show standard error.

Table 1. Effects and standard errors from linear and generalized linear models predicting donations (Model 1) and norm compliance (Models 2 and 3). Base levels for Models 1 and 3 were: Age group $=4 \& 5$; shows goodness of fit.

\begin{tabular}{llll}
\hline & $\begin{array}{l}\text { Model 1: } \\
\text { Donations }\end{array}$ & $\begin{array}{l}\text { Model 2: } \\
\text { Compliance to } \\
\text { specified norms }\end{array}$ & $\begin{array}{l}\text { Model 3: } \\
\text { Compliance to } \\
\text { equality norm }\end{array}$ \\
\hline Intercept & $2.55^{* * *}$ & -0.11 & $-1.90^{* * *}$ \\
Age group: $6 \& 7$ vs. $4 \& 5$ & $(0.31)$ & $(0.32)$ & $(0.42)$ \\
Age group: $8 \& 9$ vs. $4 \& 5$ & 0.42 & -0.63 & 0.72 \\
& $(0.28)$ & $(0.46)$ & $(0.39)$ \\
Treatment: selfish vs. control & $1.33^{* * *}$ & $-1.44^{* *}$ & $1.47^{* * *}$ \\
& $(0.29)$ & $(0.54)$ & $(0.38)$ \\
& -0.60 & & -0.53
\end{tabular}


$(0.32)$

Treatment: generous vs. control (models $1 \& 3$ ); $\quad 0.70^{*}$ generous vs. selfish (model 2)

$\mathrm{R}^{2}$

Adj. $R^{2}$

Num. obs.

AIC

BIC

Log Likelihood

Deviance
(0.32)

0.15

0.14

268

${ }^{* * *} \mathrm{p}<0.001,{ }^{* *} \mathrm{p}<0.01,{ }^{*} \mathrm{p}<0.05$

(0.43)

0.65

(0.39)

(0.63)

268

294.16

312.12

$-142.08$

284.16

\section{Did the distributions of donations vary across treatment within age groups?}

Moving beyond an analysis of means, we now turned to an analysis of the broader distribution of donations within each age and condition. Within the youngest age group, the distribution of children's donations showed variation across the norm treatments. Four- and five-year-old children who were presented with the selfish treatment made offers that were heavily skewed towards the selfish end of the spectrum when compared to children in the generous and control treatments (Fig. 2). We found differences between the selfish treatment and the control (K-S Test, $\mathrm{D}=0.6, \mathrm{~N}=56, \mathrm{p}<0.001$ ) and between the generous and selfish treatments (K-S Test, $\mathrm{D}=0.45, \mathrm{~N}=71, p=0.001$ ) but not between the generous treatment and the control $(p=0.9)$.

Within the middle age group, the distribution of donations varied to a lesser extent than in the youngest age group: offers in the selfish treatment were slightly skewed towards selfishness while offers in the generous treatment were slightly skewed towards equality (Fig. 2). Indeed, the distribution of donations varied across the generous and selfish treatments (K-S Test, $\mathrm{D}=0.4, \mathrm{~N}=76, p=0.005$ ), but not between either treatment and the control $(p s>0.3)$.

Within the oldest age group, offers in the generous treatment were skewed towards equality when compared to offers in the selfish treatment and — to a lesser extent — the control treatment. Our tests showed that indeed the distributions between the selfish and generous treatment differed (K-S Test, D $=0.45, \mathrm{~N}=$ $68, p=0.002)$. The distributions of donations between the generous treatment and the control were marginally different (K-S Test, $\mathrm{D}=0.4, \mathrm{~N}=52, p=0.055$ ), but there was not a significant difference between the distributions of donations in the selfish versus control treatments $(p=0.7)$. 


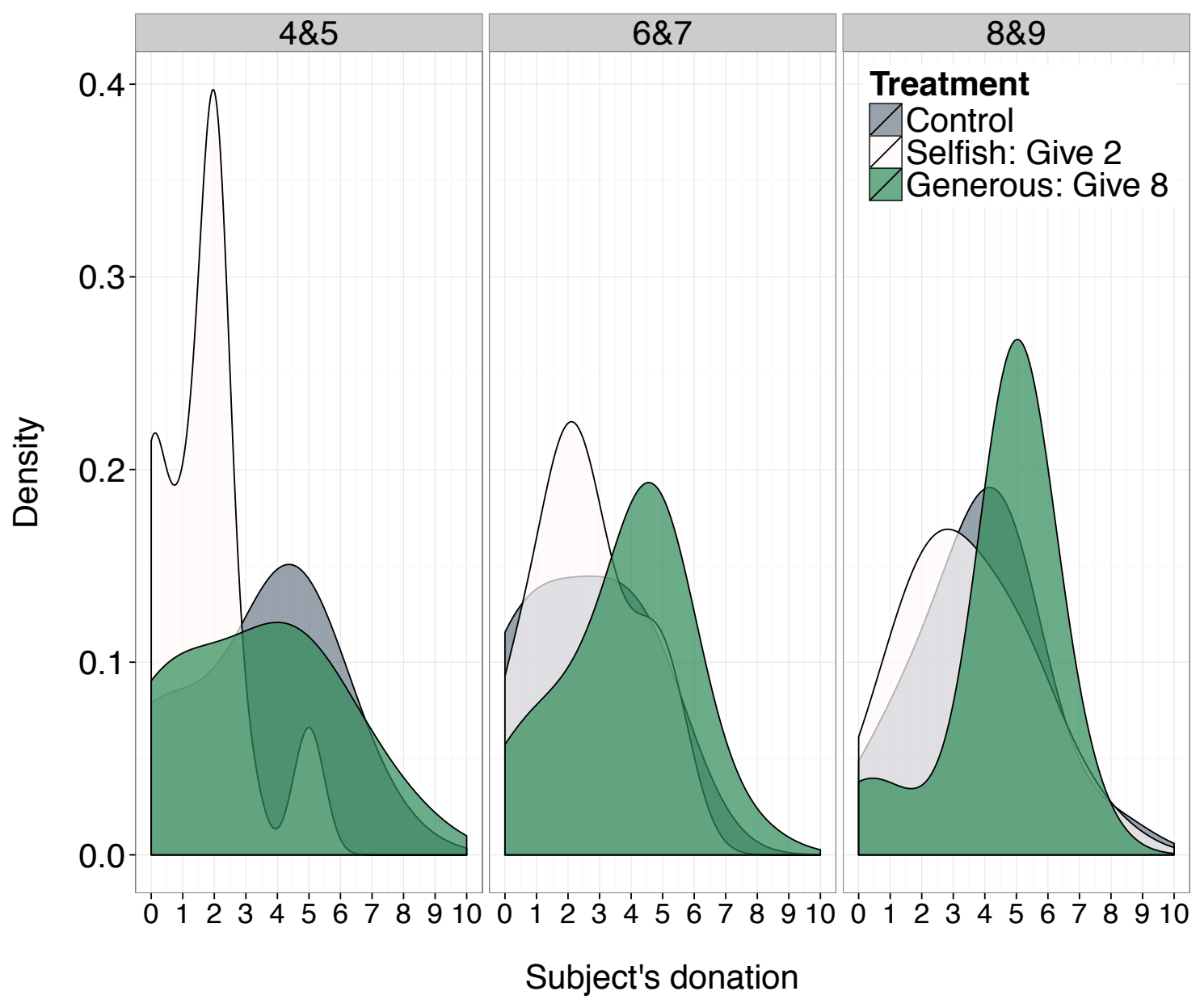

Figure 2. Density plots showing the distribution of Dictator Game donations (out of ten) across the selfish and generous treatments relative to the control condition. Density plots are faceted by age group.

\section{Were children more compliant with some norms than others?}

Our investigation of children's compliance with the specified target amounts showed that (1) younger children were generally more compliant with target norms (LRT, age group, $\chi_{2}^{2}=7.9, p=0.019$ ) and (2) children were more compliant with the selfish compared to generous norm (LRT, treatment, $\chi_{1}^{2}=38.97, p$ $<0.001$; Fig 3; Table 1). Indeed, we saw very little exact compliance with the generous norm; i.e., children rarely gave eight candies to their partner (Fig 3). Our GLMM showed no interaction between age group and treatment $(p=0.6)$. Children in the control condition were excluded from this analysis because they did not learn about a target amount. However, we conducted additional analyses to test whether receiving a norm treatment affected donations of the specified amount. To this end, we compared the frequency of exact compliance (gave exactly two, gave exactly 8 ) between children in the selfish and generous treatments compared to control children across the three age groups (Table S5). These analyses showed that, within 
the youngest age group, there was a greater frequency of children who gave exactly two candies in the treatments than in the control $\left(\chi^{2}=10.46, p=0.001\right)$.

In addition to complying to target amounts, children may have been complying with a pre-existing norm of equality (i.e., giving 5 out of 10). To investigate this, we examined whether children who received one of the norm treatments were more likely to adhere to an equality norm compared to children in the control (Figure 3, right panel). Our GLMM again showed first order effects of age group (LRT, $\chi_{2}^{2}=16.34$, $p<0.001)$ and treatment (LRT, $\left.\chi_{2}^{2}=13.16, p<0.01\right)$ but no interaction between the two $(p=0.14)$. Older children were more likely to share equally compared to younger children (Table 1). Children in the generous treatment were slightly more likely to give half than children in the control $(\beta=0.6)$ while children in the selfish treatment were slightly less likely to give half than children in the control $(\beta=-0.5)$,

361 but these were not significant effects ( $\mathrm{Ps}>0.09$; Table 1). Additionally, as above, we examined whether the frequency of compliance to an equality norm varied between children who had been exposed to a norm versus controls (Table S5). We found that, within the oldest age group, there was a marginally greater

364 frequency of children who gave exactly five candies in the generous treatment compared to the control $\left(\chi^{2}\right.$ $365=3.53, p=0.06)$. 

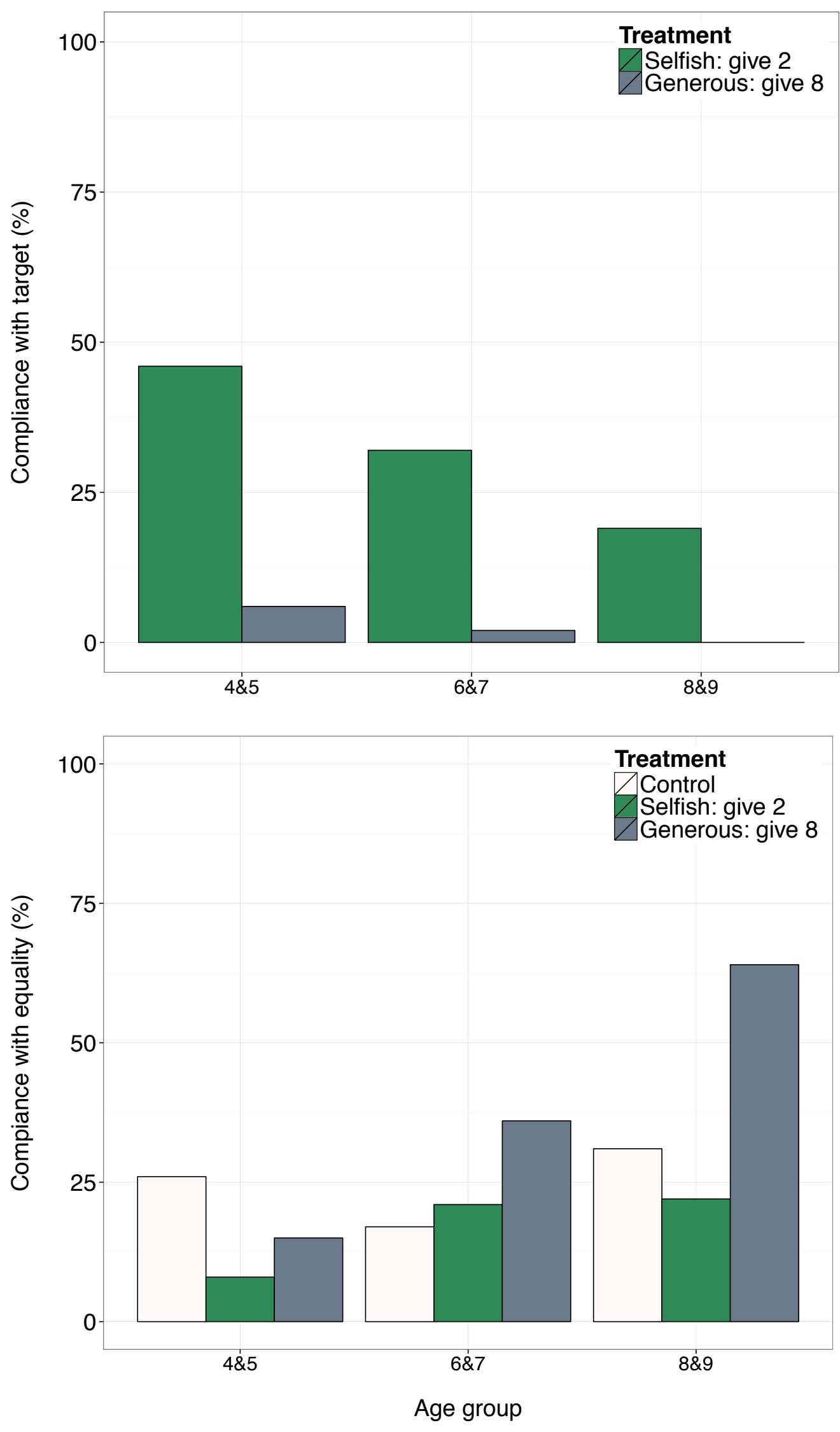
Figure 3. Percent of participants who complied exactly with the selfish or generous norms (left panel) and who complied exactly with an equality norm (right panel). Percentages are shown by participant age group and treatment. The left panel excludes participants in the control condition because they were not exposed to either norm.

\section{Discussion}

In this study we addressed two main questions. First, are children sensitive to selfish and generous norms of giving and, second, are they differentially influenced by injunctive versus descriptive information? Our results suggest that the answer to our first question is a resounding yes. Relative to their baseline giving in the control, children gave more candies to their partner when presented with the generous (give 8) norm and - to a lesser extent-gave fewer candies when presented with a selfish norm (give 2). However, contrary to predictions concerning age-related shifts in the weight accorded to different norm formats, children were not differentially influenced by either descriptive or injunctive norms. Instead, both kinds of norms moved children away from their baseline level of generosity to an equivalent degree.

Why were descriptive and injunctive norms equally effective here? One (admittedly speculative) reason for this difference could be that as children are actively internalizing the norms that govern generosity, they remain open to all sources of information about what constitutes acceptable behavior. Indeed, both peers and authority figures represent reasonable sources of such information about the way the world works, and focusing on one at the expense of the other might not be an optimal strategy. By contrast, in line with Raihani and McAuliffe (2014), adults may be more selective about which norms they choose to follow, and more sensitive to injunctive norms in particular, because they are concerned with garnering social approval or concerned about the negative consequences of violating injunctive norms (or gains in upholding them). In other words, even if many or even most people are being selfish, if one ought to be generous there may still be gains to be made from following the injunctive norm, at least in some circumstances (for example, reputational gains or the avoidance of costs associated with non-compliance).

On this account, then, children are equal-opportunity norm compliers, while adults are particularly swayed by injunctive information.

Another possibility, however, is that children simply have difficulty distinguishing descriptive from injunctive norms. Some recent research, while not directly investigating this possibility, provides enough evidence to seriously entertain it. In particular, this work suggests that children might obscure the distinction in a particular manner, namely by inferring injunctive content from descriptive information. For example, a substantial program of research (reviewed in Schmidt \& Tomasello, 2012) shows that children rapidly learn simple behavioral regularities such as how a puppet interacts with an object or the rules of a game. Despite the fact that the demonstrations from which they learn are "merely" descriptive in that no directly injunctive information is provided, children spontaneously protest subsequent deviations from the previously demonstrated behavior, suggesting that they have inferred injunctive content from the descriptive regularity. Even more generally, other recent work has shown that children readily infer 
"ought" from "is" across a wide range of examples (Tworek \& Cimpian, 2016), again suggesting a tendency to use descriptive information as evidence for injunctive information (and indeed this tendency may not go away entirely even in adults; e.g. Eidelman, Crandall, \& Pattershall, 2009). All evidence is certainly not uniformly consistent with this possibility, however. Indeed, closer to our domain of resource allocation behavior, one recent study (DeJesus, Rhodes, \& Kinzler, 2014) showed that while four and fiveyear-olds' judgments of how someone should and would distribute resources were identical, older children clearly differentiated the two kinds of judgments, in particular by thinking that others would distribute preferentially towards ingroup others even though they should distribute in line with a norm of fairness.

And some of the research we reviewed previously also suggests that children distinguish what they ought to do from what they (or a third party) will do (Smith et a., 2013; Blake et al., 2014). There are many differences across these studies that make direct comparison difficult, but we see the question of whether and when children distinguish descriptive from injunctive norms as a critical area for future investigation. This is especially true given the argument that different modes of social transmission, and by extension different norm formats, should be expected to predominate at different points of development (Hewlett et al., 2011), a view which requires that children have the capacity to distinguish them in the first place.

A more prosaic possibility for the lack of effect of norm format is that our design simply did not make the distinction between them salient enough. In the injunctive condition in our study, the Experimenter said "I think you should give $2 / 8$ ". This phrasing communicates the injunctive construction through the use of the word "should." However, this phrasing leaves open the possibility that children considered this to be the opinion of the experimenter as opposed to a norm about what ought to be done more generally. If children did consider this information to be a subjective opinion, however, we might have expected to see a stronger effect of the descriptive norm compared to the 'injunctive' norm. Instead, both norms influenced children's behavior to the same degree. Thus, we believe our injunctive phrasing successfully communicated a norm about what ought to be done generally. An interesting avenue for future work would be to test whether these norms exert differential effects in other contexts as they do in adults (Cialdini et al., 1990). For example, it is possible that these norms do not exert differential influences in the context of children's giving behavior but might be differentially effective in other contexts such as which rules can be broken (e.g. you should tidy up your toys but no-one else has tidied up their toys) and which conventions should be followed (e.g. you should tuck in your shirt but no-one else has tucked in their shirts).

Results from our distributional and compliance analyses highlighted two main age-related results. First, the distribution of children's donations varied based on whether participants were exposed to a generous or selfish norm versus no norm. Specifically, 4- and 5-year-old children's donations were particularly skewed by the selfish norm. Indeed, children in this youngest group were especially likely to show exact compliance with the selfish norm (i.e., many of them gave exactly two out of ten candies). This result is supported by our model predicting donations from only the subset of children who received the 
selfish norm versus control, which showed a significant interaction between age and donation. This finding is also supported by the results from compliance analyses, which showed that, among the youngest children, donations of exactly two candies were more common in the selfish treatment than in the control.

In contrast to the youngest age group, 8- and 9-year-old children's donations were more skewed by the generous norm. Older children who were exposed to the generous norm were especially likely to give half of their candies to their partner, showing high levels of compliance with what was perhaps a preexisting norm of equality. Yet, interestingly, these children rarely showed exact compliance with the generous norm (i.e., they almost never donated eight out of ten candies). This pattern is also supported by our contingency table analysis, which showed a trend towards more frequent equal splits in the generous condition than in the control specifically among the oldest children.

Our findings clearly show that generosity is flexible in children from early in development. However, they also demonstrate that this flexibility is constrained by pre-existing biases. Younger children, who tend to show a self-interested bias in the Dictator Game (Benenson et al., 2007), were particularly influenced by - and likely to comply exactly with - the selfish norm. This result suggests that the selfish norm had a licensing effect on their behavior; that is, 4- and 5-year-olds were more willing to be selfish if the experimenter explicitly condoned selfishness or if they learned that their peers tend to be selfish, perhaps because the match between norm and self-interest gave them cover to conform to that norm rather than a potentially competing norm of generosity or fairness which conflicted with self-interest. By contrast, older children were more influenced by the generous norm but refused to comply exactly with the target amount. Instead, the generous norm had the effect of pushing their donations towards equality. This finding is consistent with previous work that suggests that it is not until mid- to late-childhood that children begin to adhere to a norm of equality, even though younger children are aware of the norm (Blake et al., 2014; Smith et al., 2013). It is also consistent with cross-cultural work that suggests that around this period in development, children adopt adult-typical patterns of prosocial behavior (House et al., 2013). Our findings also suggest that it is not simply that younger children fail to spontaneously bring to mind a norm of which they are aware. That is, younger children's failure to comply with norms that they think should apply to others and to themselves (e.g. Smith et al., 2013) is not a limitation of failing to activate a known norm, because even when we explicitly bring a norm to mind it fails to influence their behavior. Rather, generous norms appear to be ineffective in shifting younger children's behavior even when they have been manipulated to think explicitly about it prior to acting.

Our finding that children in the generous norm condition were slightly more likely to share equally than those in the control or selfish norm condition extends past work by showing that even as children begin to move towards equality, their behavior has not become rote but rather is still susceptible to external normative influences. Interestingly, however, children did not blindly comply with stated norms. Instead, they generally capped their generosity at an equal split. Broadly, the pattern of giving in 8- and 9-year old participants suggests that with a minimal amount of information children can be persuaded to engage in 
costlier prosocial behavior than they would otherwise, but they place limits on the degree to which they accept such influence, essentially refusing to take a bad deal (i.e., to give so much that they would be left with less than half).

Children in the middle age group - the 6- and 7-year-olds-present an interesting puzzle. According to our distributional analyses, these children were not influenced by either the selfish or generous norms compared to the control. However, the distribution of children's responses did differ across selfish and generous treatments: offers were skewed towards selfishness in the selfish treatment and towards equality in the generous condition. These findings suggest that both norms exerted some influence, though to a lesser extent than in both younger and older children. While future work is necessary to understand why normative information was not as influential in this age range, we tentatively suggest that this is because 6- and 7-year-olds are in a transition stage, moving away from a self-focused default to a more egalitarian default; this state of more active internal conflict leads them to be less readily licensed by the selfish norm (as compared to younger children) but also less willing to give away a full half of their rewards (as compared to older children). In other words, for children in this age range, the pull of the generous norm is attenuated by a pre-existing desire to keep slightly more for oneself while the pull of the selfish norm is attenuated by the knowledge that one ought to share equally.

In sum, our results demonstrate that children are sensitive to information that communicates norms of giving. However, this sensitivity shows age-related change, with younger children more influenced by selfishness and older children more influenced by generosity. Further, children's receptivity to new normative information is constrained by their pre-existing biases, potentially including previously acquired norms of fairness. More broadly, our results begin to shed light on the ontogeny of giving behavior, and in particular its flexible tuning to local norms. Both information about what others are doing as well as information about what ought to be done likely contribute to the emerging differences in how children and adults engage in costly giving behavior. 


\section{Acknowledgements}

501 We are grateful to Peter Blake for sharing his Dictator Game script, which we adapted for this study, and 502 for his feedback on an earlier version of this manuscript. We are also grateful to Shaina Coogan, Terri 503 Frasca and the many research assistants who helped collect, enter, and code these data. We would 504 additionally like to thank three anonymous reviewers for their helpful comments on this manuscript as well 505 as the Greater Good Science Center at UC Berkeley and the John Templeton Foundation for support. 


\section{References}

Benenson, J. F., Pascoe, J., \& Radmore, N. (2007). Children's altruistic behavior in the dictator game. Evolution and Human Behavior, 28(3), 168-175.

Bicchieri, C., \& Xiao, E. (2009). Do the right thing: but only if others do so. Journal of Behavioral Decision Making, 22, 191-208.

Blake, P. R., \& Rand, D. G. (2009). Currency value moderates equity preference among young children. Evolution and Human Behavior, 31, 210-218.

Blake, P. R., Piovesan, M., Montinari, N., Warneken, F., \& Gino, F. (2015). Prosocial norms in the classroom: The role of self-regulation in following norms of giving. Journal of Economic Behavior \& Organization, 115, 18-29.

Blake, P. R., Corbit, J., Callaghan, T. C., \& Warneken, F. (2016). Give as I give: Adult influence on children's giving in two cultures. Journal of Experimental Child Psychology, 152, 149-160.

Brown, B. B. (1990). Peer groups and peer cultures. In S. S. Feldman and G. R. Elliott (Eds.), At The Threshold: The Developing Adolescent. Cambridge, MA: Harvard University Press, pp 171-196.

Camerer, C. F. (2003). Behavioral Game Theory. Princeton, NJ: Princeton University Press.

Cavalli-Sforza, L. L., \& Feldman, M. W. (1981). Cultural transmission and evolution: a quantitative approach. Princeton, NJ: Princeton University Press.

Cialdini, R. B., Demaine, L. J., Sagarin, B. J., Barrett, D. W., Rhoads, K., \& Winter, P. L. (2006). Managing social norms for persuasive impact. Social influence, 1, 3-15.

Cialdini, R. B., Reno, R. R., \& Kallgren, C. A. (1990). A focus theory of normative conduct: recycling the concept of norms to reduce littering in public places. Journal of Personality and Social Psychology, 58, 1015.

Cowell, J. M., Lee, K., Malcolm Smith, S., Selcuk, B., Zhou, X., \& Decety, J. (2016). The development of generosity and moral cognition across five cultures. Developmental science, 1-12.

DeJesus, J. M., Rhodes, M., \& Kinzler, K. D. (2014). Evaluations Versus Expectations: Children's Divergent Beliefs About Resource Distribution. Cognitive Science, 38(1), 178-193. http://doi.org/10.1111/cogs.12093

Eidelman, S., Crandall, C. S., \& Pattershall, J. (2009). The existence bias. Journal of Personality and Social Psychology, 97(5), 765-775.

Engel, C. (2011). Dictator games: a meta study. Experimental Economics, 14(4), 583-610.

Fehr, E., Bernhard, H., \& Rockenbach, B. (2008). Egalitarianism in young children. Nature, 454(7208), $1079-1083$.

Gummerum, M., Hanoch, Y., Keller, M., Parsons, K., \& Hummel, A. (2010). Preschoolers' allocations in the dictator game: The role of moral emotions. Journal of Economic Psychology, 31(1), 25-34.

Henrich, J., Boyd, R., Bowles, S., Camerer, C., Fehr, E., Gintis, H., et al. (2005). "Economic man” in crosscultural perspective: behavioral experiments in 15 small-scale societies. The Behavioral and Brain Sciences, 28, 795-815; discussion 815-55.

Hewlett, B. S., Fouts, H. N., Boyette, A. H., \& Hewlett, B. L. (2011). Social learning among Congo Basin hunter-gatherers. Philosophical Transactions of the Royal Society of London B: Biological Sciences, 366, 1168-1178. 
House, B. R., Henrich, J., Brosnan, S. F., \& Silk, J. B. (2012). The ontogeny of human prosociality: behavioral experiments with children aged 3 to 8. Evolution and Human Behavior, 33, 291-308.

House, B. R., Silk, J. B., Henrich, J., Barrett, H. C., Scelza, B. A., Boyette, A. H., et al. (2013). Ontogeny of prosocial behavior across diverse societies. Proceedings of the National Academy of Sciences, 110, 14586-14591.

Jaswal, V. K., \& Neely, L. A. (2006). Adults don't always know best preschoolers use past reliability over age when learning new words. Psychological Science, 17, 757-758.

Kahneman, D., Knetsch, J.L., Thaler, R.H. (1986). Fairness and the assumptions of economics, Journal of Business, 59, S285-S300.

Moore, C. (2009). Fairness in Children's Resource Allocation Depends on the Recipient. Psychological Science, 20, 944-948.

Raihani, N. J., \& McAuliffe, K. (2014). Dictator Game Giving: The importance of descriptive versus injunctive norms. PLoS ONE, 9(12), e113826.

Rakoczy, H., Hamann, K., Warneken, F., \& Tomasello, M. (2010). Bigger knows better: Young children selectively learn rule games from adults rather than from peers. British Journal of Developmental Psychology, 28, 785-798.

Rakoczy, H., \& Schmidt, M. F. (2012). The early ontogeny of social norms. Child Development Perspectives, 7, 17-21.

Rakoczy, H., Warneken, F., \& Tomasello, M. (2008). The sources of normativity: young children's awareness of the normative structure of games. Developmental Psychology, 44, 875.

Rochat, P., Dias, M. D., Liping, G., Broesch, T., Passos-Ferreira, C., Winning, A., \& Berg, B. (2009). Fairness in distributive justice by 3 -and 5-year-olds across seven cultures. Journal of CrossCultural Psychology, 40, 416-442.

Schmidt, M. F. H., \& Tomasello, M. (2012). Young Children Enforce Social Norms. Current Directions in Psychological Science, 21(4), 232-236.

Schmidt, M. F. H., Rakoczy, H., \& Tomasello, M. (2012). Young children enforce social norms selectively depending on the violator's group affiliation. Cognition, 124(3), 325-333.

Schultz, P. W., Nolan, J. M., Cialdini, R. B., Goldstein, N. J., \& Griskevicius, V. (2007). The constructive, destructive, and Reconstructive power of social norms. Psychological Science, 18(5), 429-434.

Sekhon, J. (2011). Multivariate and propensity score matching software with automated balance optimization: The Matching package for R. Journal of Statistical Software, 42(7), 1-52.

Smith, C. E., Blake, P. R., \& Harris, P. L. (2013). I should but I won't: why young children endorse norms of fair sharing but do not follow them. PLOS ONE, 8(3), e59510.

Tworek, C. M., \& Cimpian, A. (2016). Why Do People Tend to Infer "Ought" From "Is?" The Role of Biases in Explanation. Psychological Science, 27(8), 1109-1122. 